\title{
Neural mechanisms supporting flexible performance adjustment during development
}

\author{
Eveline A. Crone \\ Leiden University, Leiden, The Netherlands \\ and Leiden Institute for Brain and Cognition, Leiden, The Netherlands \\ KIKI ZANOLIE \\ Leiden University, Leiden, The Netherlands \\ Leiden Institute for Brain and Cognition, Leiden, The Netherlands \\ and Erasmus University Rotterdam, Rotterdam, The Netherlands \\ Linda Van LeiJenhorst and P. Michiel Westenberg \\ Leiden University, Leiden, The Netherlands \\ and Leiden Institute for Brain and Cognition, Leiden, The Netherlands \\ AND \\ SERGe A. R. B. Rombouts \\ Leiden University, Leiden, The Netherlands \\ Leiden Institute for Brain and Cognition, Leiden, The Netherlands \\ and Leiden University Medical Center, Leiden, The Netherlands
}

\begin{abstract}
Feedback processing is crucial for successful performance adjustment following changing task demands. The present event-related fMRI study was aimed at investigating the developmental differences in brain regions associated with different aspects of feedback processing. Children age 8-11, adolescents age 14-15, and adults age 18-24 performed a rule switch task resembling the Wisconsin Card Sorting Task, and analyses focused on different types of negative and positive feedback. All age groups showed more activation in lateral orbitofrontal cortex (OFC), anterior cingulate cortex (ACC), dorsolateral prefrontal cortex (DLPFC), and superior parietal cortex following negative relative to positive performance feedback, but the regions contributed to different aspects of feedback processing and had separable developmental trajectories. OFC was adultlike by age 8-11, whereas parietal cortex was adultlike by age 14-15. DLPFC and ACC, in contrast, were still developing after age 14-15. These findings demonstrate that changes in separable neural systems underlie developmental differences in flexible performance adjustment. Supplementary data from this study are available online at the Psychonomic Society Archive of Norms, Stimuli, and Data, at www.psychonomic.org/archive.
\end{abstract}

In a changing environment, it is of great importance to flexibly adjust behavior on the basis of information about personal performance. An essential component of flexible behavioral adjustment is the ability to use performance feedback for subsequent performance. Feedback monitoring has been studied in the neuropsychological literature using the well-known Wisconsin Card Sorting Task (WCST; Demakis, 2003; Milner, 1963). This task requires participants to sort cards by color, shape, or number of items according to sorting rules the participants do not know beforehand. After a number of correct consecutive sorts, the sorting rule changes without warning, and participants need to infer the new rule on the basis of positive and negative feedback. The advantage of this task is that it requires monitoring of performance errors and flexible performance adjustment, thereby resembling real-life sit- uations in which performance also needs to be adjusted on the basis of changing environmental demands.

Poor WCST performance has been associated with damage to lateral prefrontal cortex (lat-PFC). Patients with lat-PFC damage have been found to perseverate in applying the previously correct sorting rule (Barceló \& Knight, 2002). Behavioral studies show that 6 - to 12 -year-old children also have difficulty performing the WCST because their performance resembles that of patients with lat-PFC damage (Huizinga, Dolan, \& van der Molen, 2006; Welsh, Pennington, \& Groisser, 1991). The protracted development of performance monitoring has been confirmed in other paradigms in which correct performance was dependent on feedback processing (Luciana \& Nelson, 1998). Recently, a number of electrophysiological studies have investigated how children and adolescents monitor

E.A. Crone, ecrone@fsw.leidenuniv.n 
their performance after committing errors. Using speeded flanker or go/no-go tasks, researchers have studied developmental differences in a negative scalp deflection that is time-locked to an incorrect response (the error-related negativity [ERN]; Falkenstein, Hohnsbein, Hoormann, \& Blanke, 1991). Importantly, this potential is sensitive to committing an error even without performance feedback. Developmental studies have demonstrated that error monitoring, as indexed by the size of the ERN, continues to develop into adolescence (Davies, Segalowitz, \& Gavin, 2004; Kim, Iwaki, Imashioya, Uno, \& Fujita, 2007; Wiersema, van der Meere, \& Roeyers, 2007). Thus, behavioral studies have demonstrated developmental changes in feedback learning, and event-related potential (ERP) researchers have demonstrated changes in error monitoring until late adolescence. This protracted developmental trajectory has great implications for learning, because most of the improvements are based on the evaluation of internal or external feedback about one's own behavior. It is currently not known which brain regions underlie the development of performance monitoring.

Neuroimaging studies involving adult participants have implicated orbitofrontal cortex (OFC), anterior cingulate cortex (ACC), and lat-PFC in feedback processing and have provided evidence that these areas are important for signaling changing task demands, hypothesis testing, and error monitoring (Mars et al., 2005; Monchi, Petrides, Petre, Worsley, \& Dagher, 2001; O'Doherty, Critchley, Deichmann, \& Dolan, 2003). Lateral OFC (lat-OFC; in particular, Brodmann's area [BA] 47) has been found to be sensitive to feedback indicating losses, suggesting that this region responds to the emotional component of negative feedback (Frank \& Claus, 2006; Knutson, Fong, Bennett, Adams, \& Hommer, 2003; O'Doherty et al., 2003). Prior studies have indicated that lat-OFC is hypersensitive to feedback indicating loss of money in 8- to 12-year-olds (Van Leijenhorst, Crone, \& Bunge, 2006). In addition, research in patients has shown that OFC damage leads to impairments in future orientation (Bechara, Damasio, \& Damasio, 2000) or in reward/punishment processing (Rolls, 2004). Furthermore, animal studies have indicated that OFC damage results in impulsive behavior when learning from feedback (Walton, Devlin, \& Rushworth, 2004). Together, these studies suggest that OFC is critical for emotional evaluation of performance outcomes.

Like lat-OFC, dorsal ACC (BAs 24 and 32) has been found to be recruited more following negative rather than positive feedback (Holroyd et al., 2004; Mars et al., 2005), but this effect has only been observed under certain task demands (Nieuwenhuis, Slagter, von Geusau, Heslenfeld, \& Holroyd, 2005; van Veen, Holroyd, Cohen, Stenger, \& Carter, 2004). Specifically, ACC is most active following unexpected negative feedback in situations in which feedback is dependent on a choice between two or more alternatives. This feedback-related response has been interpreted as sensitivity to the first information showing that outcomes are worse than anticipated (Holroyd \& Coles, 2002) or to feedback indicating uncertainty (Walton et al., 2004).

Within lat-PFC, dorsolateral PFC (DLPFC) has been linked to the informative value of feedback with respect to goal-directed actions (Miller \& Cohen, 2001; Zanolie, Van Leijenhorst, Rombouts, \& Crone, 2008). In the WCST, feedback can be used to infer information about the correctness of a certain behavior rule. DLPFC has been found to be sensitive to feedback that shows whether a tested hypothesis is correct or incorrect (Zanolie et al., 2008). Prior developmental fMRI studies have demonstrated pronounced changes in DLPFC's activation profile in a working memory task (Crone, Wendelken, Donohue, Van Leijenhorst, \& Bunge, 2006). Interestingly, agerelated changes in activation of DLPFC co-occur with changes in activation in superior parietal cortex (Crone, Wendelken, et al., 2006; Klingberg, Forssberg, \& Westerberg, 2002). This result is consistent with findings indicating that DLPFC and superior parietal cortex show the slowest structural changes in gray matter maturation (Gogtay et al., 2004). The monkey literature suggests that the parietal cortex is part of a parietofrontal network (Rizzolatti, Luppino, \& Matelli, 1998), and human imaging studies have reported activation in parietal cortex in other high-order cognitive paradigms, including stimulus and response selection tasks (Bunge, Hazeltine, Scanlon, Rosen, \& Gabrieli, 2002; Liston, Matalon, Hare, Davidson, \& Casey, 2006). However, the role of superior parietal cortex in feedback processing is currently unknown.

In the present study, we sought to examine whether developmental changes in feedback processing in a rule-shift task resembling the WCST could be accounted for by immaturity of lat-OFC, ACC, DLPFC, or superior parietal cortex. Developmental changes are observed in error monitoring until late adolescence, but different processes and associated neural circuitry may account for these differences. Thus, we examined feedback sensitivity in childhood (8-11 years), adolescence (14-15 years), and adulthood (18-25 years) using a child-friendly WCST-like feedback monitoring task (Crone, Somsen, Zanolie, \& van der Molen, 2006) previously shown to be sensitive to process dissociability in the areas under study (Zanolie et al., 2008).

Figures 1 and 2 give schematic presentations of the task and conditions under study. Participants were instructed to help a dog find its way back home by pressing one of four response buttons that corresponded to four locations on the screen. Each response was followed by a visually presented feedback sign that could be positive $(+)$ or negative $(-)$ (see Figure 1A). Participants were taught three possible response rules (A, B, and C in Figure 1B; see the Method section for further details). During the task, we had participants switch between the three spatial compatibility rules that were taught prior to scanning. However, the switch occurred unexpectedly and without warning, and the correct rule had to be inferred on the basis of positive and negative feedback.

We dissociated three types of negative feedback and two types of positive feedback. These feedback types are illustrated in Figure 2. Negative feedback could indicate that the previously applied rule was no longer correct (first warning feedback), that the tested rule during rule searching was incorrect (efficient negative feedback), or that an error was committed during rule application (error feedback). The first warning negative feedback gave the first 
A

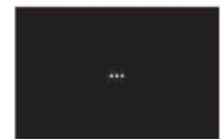

$500 \mathrm{msec}$

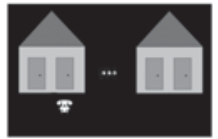

$2,500 \mathrm{msec}$

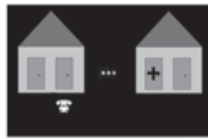

$1,000 \mathrm{msec}$

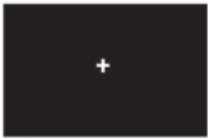

Jitter

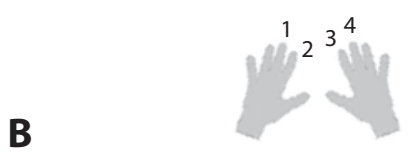

B

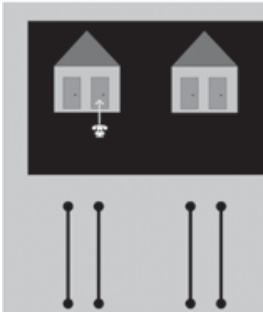

Rule A

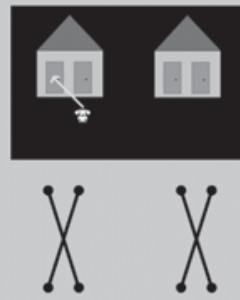

Rule B

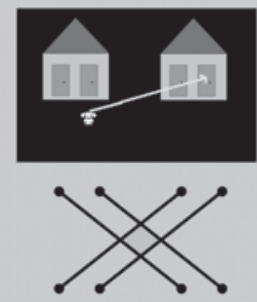

Rule C

Figure 1. Display of task sequence (A) and rule types (B). Participants were told to infer one of the spatial mapping rules that were trained prior to scanning. The task was changed into a prosocial game by explaining to the participants that they should help the dog find its way back home. The dog could appear in one of four locations, and the participants were instructed to open one of the four doors (locations) by pressing the corresponding response key. Their selection was followed by a visually presented feedback sign $(+$ or -$)$. The rules changed unannounced following two, three, or four consecutive correct sorts. The spatial mapping rules are displayed in the bottom part of panel $B$ (see the text for explanations).

indication that an applied response rule had to be switched (e.g., in Figure 2A, that Rule A is no longer correct). This feedback violated the participant's expectation because it occurred after a series of correct responses. Following the first warning feedback, the participant had a $50 \%$ chance of applying the correct rule (Rule B or C). In case the incorrect rule was applied following a first warning negative feedback (here, Rule B), the participants knew with 100\% certainty what the correct rule had to be (here, Rule C). Therefore, the feedback that followed the application of the incorrect rule (here, Rule B) was referred to as an efficient negative feedback, because it was successfully used to test a hypothesis about the possible correct rule. When an erroneous response was given within a series of correct responses (Figure 2B), this resulted in negative feedback that was referred to as an error negative feedback. This was an error negative feedback because the participant made an error while knowing the correct response rule. The latter feedback type is most likely associated with several control processes, including expectation violations, hypothesis testing, and uncertainty. Positive feedback could give the first indication that the correct rule was found after rule searching (first positive feedback) or that the correct rule was applied (correct positive feedback).

In a prior study that included only adults, we demonstrated that DLPFC and ACC were both most active following error negative feedback, suggesting that both regions are important for error monitoring. However, the regions were differentially sensitive to first warning and efficient negative feedback. That is, DLPFC was more sensitive to efficient negative feedback and first positive feedback (i.e., feedback that was used for hypothesis testing), whereas ACC was more sensitive to first warning feedback (i.e., feedback that signaled a violation of expectations; Zanolie et al., 2008). The objective of the present eventrelated fMRI study was to test the neurodevelopmental trajectories of lat-OFC, ACC, DLPFC, and superior parietal cortex when participants needed to flexibly switch between rules in response to positive and negative feedback.

To date, no published study has investigated the developmental correlates of feedback processing with fMRI. However, we previously examined the development of feedback processing in children ages $8-10$ and $11-12$ and in adults in rule-learning paradigms using heart rate measures (Crone, Jennings, \& van der Molen, 2004; Crone, Somsen, et al., 2006). These studies demonstrated that the presentation of negative feedback is associated with slowing of the subsequent heartbeat. Importantly, in adults this slowing only occurred for feedback that was informative for subsequent behavioral adjustment, not for feedback that was unrelated to performance (e.g., random feedback; Crone et al., 2003). In contrast, 8- to 10-year-olds showed heart rate slowing to all negative feedback, whether it was informative or uninformative for behavior, suggesting that they failed to discriminate the informative value of feedback when learning rules (Crone, Jennings, \& van der Molen, 2004). These 


\section{Example 1}

\begin{tabular}{|c|c|c|c|c|c|}
\hline $\begin{array}{c}\text { Correct rule } \\
\text { (changes without warning) }\end{array}$ & A & $C$ & C & C & C \\
\hline \multirow[b]{2}{*}{$\begin{array}{l}\text { Applied Rule and FB } \\
\text { Feedback Type }\end{array}$} & & $\stackrel{50 \%}{\longrightarrow}$ & $\begin{array}{c}\mathbf{B}=- \\
\text { Efficient FB }\end{array}$ & $\begin{array}{c}\mathbf{C}=+ \\
\text { First positive FB }\end{array}$ & \\
\hline & $\begin{array}{c}\text { A }=++ \\
\text { Correct FB }\end{array}$ & $\begin{array}{l}\quad A=- \\
\text { First warning FB }\end{array}$ & & & $\begin{array}{c}\mathbf{C}=+ \\
\text { Correct FB }\end{array}$ \\
\hline & & $\underset{50 \%}{\longrightarrow}$ & $\begin{array}{l}\quad \mathbf{C}=+ \\
\text { First positive FB }\end{array}$ & $\begin{array}{c}\mathrm{C}=+ \\
\text { Correct FB }\end{array}$ & \\
\hline
\end{tabular}

\section{Example 2}

\begin{tabular}{|c|}
\hline Correct rule \\
(changes without warning) \\
\hline
\end{tabular}

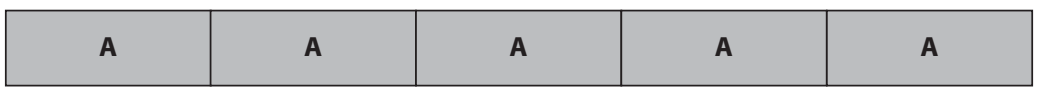

Applied Rule and FB
Feedback Type

\begin{tabular}{|c|c|c|}
\hline $\begin{array}{c}\mathbf{A}=+ \\
\text { Correct FB }\end{array}$ & $\begin{array}{c}\mathbf{A}=+ \\
\text { Correct FB }\end{array}$ & $\begin{array}{c}\mathbf{B}=- \\
\text { Error FB }\end{array}$ \\
\hline
\end{tabular}

$\mathbf{A}=+$
Correct FB

$\mathbf{A}=+$
Correct FB

Figure 2. Examples of feedback (FB) types. Three negative feedback types (first warning, efficient, and error) and two positive feedback types (first and correct) were determined post hoc for each individual separately. In the top row, the currently active response rule is displayed (as selected by the computer), which could be one out of three rules (referred to as $\mathrm{A}, \mathrm{B}, \mathrm{C}$ ). These rules were pretrained, but it was not known by the participants which rule was currently correct. The correct rule had to be inferred on the basis of positive and negative feedback. The second row shows examples of the applied response rule and the feedback that followed this response rule. Example 1 shows a correct rule use (Rule A), followed by a first warning negative feedback (A is no longer correct). Following the first warning feedback, the participant tries one of the two other possible response rules (Rule B or C). This selection results in first positive feedback on $50 \%$ of the trials (here, Rule C) and in negative feedback on the other $50 \%$ of the trials (here, Rule B). If the negative feedback is followed by application of the correct response rule on the next trial (here, Rule $\mathrm{C}$ ), this feedback is referred to as an efficient negative feedback, because it was used to find the correct rule. Example 2 shows an erroneous response in a series of correct responses. This type of negative feedback is referred to as an error negative feedback.

findings made us hypothesize that neurodevelopmental changes in feedback processing would be most pronounced in ACC and DLPFC, regions that are sensitive to signaling the informative value of feedback.

\section{METHOD}

\section{Participants}

Twenty adults age $18-24$ (mean age 20.3 years; 8 men, 12 women), 20 adolescents age $14-15$ (mean age 14.5 years; 11 boys, 9 girls), and 17 children age $8-11$ (mean age 9.5 years; 9 boys, 8 girls) were included in the study. Participants were recruited through local advertisements and from Leiden University in the Netherlands. Participants' consent was obtained according to the Declaration of Helsinki, and the study was approved by the Internal Review Board at Leiden University Medical Centre. Three additional children age 8-11 were excluded because of excessive head movement $(>2 \mathrm{~mm}$ of translation in any direction) or failure to perform the task. A chi-square analysis indicated that the proportions of men and women did not differ between age groups $\left[\chi^{2}(2)=0.62, p=.73\right]$. The adult data have been published separately as evidence for the functional dissociation between different types of feedback processing (Zanolie et al., 2008).

\section{Behavioral Assessment}

Both children and adults participated in a separate behavioral testing session before scanning. Cognitive functioning was assessed using the Similarities and Blocks Design subtests from the Wechsler Intelligence Scale for Adults and the Wechsler Intelligence Scale for Children. Estimated IQs were 111 for 8- to 11-year-olds, 104 for 14- to 15 -year-olds, and 105 for 18 - to 24 -year-olds. The differences in IQ between groups were significant $[F(1,32)=9.09, p<.001]$, but all IQ scores were in the normal range. Children and adults practiced the behavioral tasks in a quiet laboratory. Children were also trained to lie still in a mock scanner, which simulated the environment and sounds of an actual MRI scanner. Parents filled out behavioral questionnaires in this session. Participants were screened for psychiatric conditions with the Child Behavior Checklist for children age 8-17 (Achenbach, 1991) or the Symptoms Checklist-Revised for the adults, age 18-25 years. All participants had scores within $1 S D$ of the mean of a normative standardized sample.

\section{Experimental Task}

The task used in the scanner was meant to manipulate demands on the processing of feedback when the participant flexibly changed between rules. The task was a child-friendly rule-switch task that was used in prior behavioral research and was adapted for fMRI purposes (Crone, Jennings, \& van der Molen, 2004). Participants were asked to respond to a stimulus that could appear in one of four horizontally presented locations on the screen by pressing appropriate buttons (see Figure 1). Prior to scanning, participants were trained to use one of three spatial stimulus-response rules. The four possible answers were mapped to the four buttons that were mapped to the index and middle fingers from the left and right hand (see Figure 1A). Following Rule A, stimuli that appeared in one of the four locations designated a response with the finger compatible to the location. Thus, spatially compatible responses were required in response to the location of the stimulus. Following Rule B, stimuli that appeared in any of the four locations designated a response with the opposite finger of the same 
hand. Following Rule C, stimuli that appeared in any of the four locations designated a response with the finger that was assigned to the location two positions from the stimulus location (see Figure 1B). In a prior behavioral study, we found that the incompatible rules were more difficult than the compatible rule, but this difference was similar across age groups (Crone, Jennings, \& van der Molen, 2004).

On each trial, a 2.5-sec stimulus display was presented that required a buttonpress. If a response was not made within $2.5 \mathrm{sec}$, a warning was presented indicating that the response was too slow and that faster responses were required on the next trial. These trials were not included in the analysis and consisted of less than $2 \%$ of the trials. Following the stimulus display and the response, a 1.5-sec feedback display was visually presented, with a positive or negative feedback sign (see Figure 1).

To familiarize participants with the stimuli and rules, each rule was trained in separate blocks. Our training program required a baseline success on $80 \%$ of the trials for participants to start the new practice trials with a minimum set of 15 trials. The order of the practice rules was counterbalanced between participants. The symbolic meanings of positive and negative feedback were explained, and all participants understood the meanings.

After practicing each rule separately, participants continued with a training of the task in which rules were mixed. During this phase, the critical response rule was unknown to the participants and had to be inferred using trial-to-trial feedback. Participants were told that the correct rule could change from time to time, and they were instructed to then use trial-to-trial feedback to infer the new response rule. When participants had correctly applied the response rule for 2-4 consecutive trials, the rule changed without warning. The practice task consisted of 100 intermixed trials. The task was then presented in the scanner in three blocks of 100 trials each, leading to a total of 300 trials. The three blocks that had to be completed in the scanner were the same as the mixed practice block. Before each fMRI scan, the experimenter reminded the participants of the task requirements.

\section{Feedback Scoring}

The five feedback types were determined post hoc for each individual separately. Their definitions were as follows. (1) First warning negative feedback was the first negative feedback that followed a successfully completed sequence of rule applications. This negative feedback was given unannounced once the rule had been correctly applied on two, three, or four trials (randomly determined for each rule separately); it indicated that the previously applied response rule was no longer correct, and thus indicated a rule switch. (2) Efficient negative feedback indicated that the rule chosen when searching for the appropriate rule was incorrect. When, for example, the participant was applying Rule $\mathrm{A}$ and received a first warning feedback, Rule B or $\mathrm{C}$ should be applied on the next trial. Thus, the participant had a $50 \%$ chance of applying the correct rule after the switch. When the incorrect rule was chosen first - for example, Rule B - the participant then knew which rule to apply on the next trial (Rule C). Thus, the efficient negative feedback was used to find the right response rule. (3) Error negative feedback trials consisted of those trials in which the participants failed to apply the correct response when the response rule had not changed. These three negative feedback types were contrasted with two types of positive feedback: (4) First positive feedback indicated that the correct rule had been found following a rule switch, and (5) correct positive feedback indicated correct rule use. For examples of the different types of feedback, see Figure 2.

\section{Data Acquisition}

Trials were presented in three scans of 8.2 min each. During scanning, 300 trials were presented and the rules were switched in pseudorandomized order. The order of trials within each scan was determined by using an optimal sequencing program designed to maximize efficiency of recovery of the blood oxygenation level dependent (BOLD) response (Dale, 1999). Jitter varied from 2 to $8 \mathrm{sec}$.

Scanning was performed with a standard whole-head coil on a 3.0 Tesla Philips scanner at the Leiden University Medical Center.
Functional data were acquired using T2*-weighted echo-planar imaging (EPI) during three functional runs of 232 volumes each, of which the first 2 volumes were discarded in order to allow for equilibration of T1 saturation effects $(\mathrm{TR}=2.211 \mathrm{sec}, \mathrm{TE}=30 \mathrm{msec}$, ascending interleaved acquisition, 38 slices of $2.75 \mathrm{~mm}$, field of view $220 \mathrm{~mm}, 80 \times 80$ matrix, in-plane resolution $2.75 \mathrm{~mm}$ ). Highresolution T1-weighted anatomical images were also collected after the functional runs. Head motion was restricted using a pillow and foam inserts that surrounded the head. Visual stimuli were projected onto a screen that was viewed through a mirror.

\section{fMRI Data Analysis}

Data were preprocessed using SPM2 (Wellcome Department of Cognitive Neurology, London). Images were corrected for differences in timing of slice acquisition, followed by rigid-body motion correction. Functional volumes were spatially normalized to EPI templates. The normalization algorithm used a 12-parameter affine transformation with a nonlinear transformation involving cosine basis functions, and then resampled the volumes to $3-\mathrm{mm}$ cubic voxels. Templates were based on the MNI305 stereotaxic space (Cocosco, Kollokian, Kwan, \& Evans, 1997), an approximation of Talairach space (Talairach \& Tournoux, 1988). Functional volumes were spatially smoothed with an 8-mm full-width-at-half-maximum isotropic Gaussian kernel. Statistical analyses were performed on individual participants' data using the general linear model in SPM2. The fMRI time series data were modeled by a series of events convolved with a canonical hemodynamic response function (HRF). The feedback stimulus of each trial was modeled as an event of interest. The trial functions were used as covariates in a general linear model, along with a basic set of cosine functions to high-pass filter the data, as well as a covariate for session effects. The least-squares parameter estimates of the height of the best-fitting canonical HRF for the different conditions were used in pairwise contrasts. The resulting contrast images (negative vs. positive feedback), computed on a participant-by-participant basis, were submitted to group analyses. At the group level, contrasts between conditions were computed by performing one-tailed $t$ tests on these images, treating participants as a random factor. Task-related responses were considered significant if they consisted of at least 10 contiguous voxels that exceeded either a corrected threshold of $p<$ .05 or an uncorrected threshold of $p<.001$ (both reported).

Region-of-interest (ROI) analyses were performed with the MarsBaR toolbox in SPM2 (Brett, Anton, Valabregue, \& Poline, 2002; available at marsbar.sourceforce.net). ROIs that spanned several functional brain regions were subdivided by sequentially masking the functional ROI with each of several anatomical MarsBaR ROIs. We made use of the anatomical template ROIs included with the MarsBaR program. DLPFC, ACC, lat-OFC, and superior parietal cortex ROIs were created from activation clusters for the contrast all feedback conditions $>$ fixation at $p<.001$ (uncorrected) across all participants. For all ROI analyses, effects were considered significant at an $\alpha$ of .05. For each ROI, the center of mass is reported.

\section{RESULTS}

\section{Performance}

Performance differences were examined by comparing the numbers of feedback observations over the course of 300 trials. An age group (3 levels: $8-11$ years, $14-15$ years, 18-24 years) $\times$ feedback type ( 5 levels: first warning feedback, efficient negative feedback, error negative feedback, first positive feedback, correct feedback) ANOVA confirmed that the age groups differed in number of feedback observations $[F(8,216)=19.52, p<.001$; see Figure 3]. Comparisons for each feedback type separately showed that 14- to 15-year-old adolescents did not differ from adults in number of feedback observations, except in the number of errors, which was marginally higher for adoles- 
cents than for adults $[F(1,38)=3.10, p=.08]$. In contrast, 8 - to 11-year-old children had fewer first warning feedbacks, fewer efficient feedbacks, more errors, and fewer first positive feedbacks than did adolescents and adults (all $p$ s $<.01)$. Children did not differ from adolescents and adults in number of correct feedback observations.

An additional analysis focused specifically on number of perseverative errors. Errors were scored as perseverative when the rule from the previous trials was repeated on the trial following the first warning feedback (Heaton, Chelune, Talley, Kay, \& Curtiss, 1993). An age groups (3) ANOVA for number of perseverative errors revealed significant group differences $[F(2,54)=9.86, p<.001]$, showing that children made more perseverative errors $($ mean $=7.6)$ than did adolescents $($ mean $=4.1)$ and adults $($ mean $=2.9)$. The latter two age groups did not differ from each other.

A second set of analyses examined reaction time (RT) differences between the age groups. For this analysis, RTs preceding error negative feedback were compared with those preceding correct feedback. We performed this analysis to examine whether participants were uncertain about their responses on error trials relative to correct trials. A two-way ANOVA showed that RTs differed between conditions and age groups [age group $\times$ feedback type interaction: $F(2,54)=4.90, p<.01]$. Separate comparisons for each age group revealed that adults were slower on error negative feedback $(962 \mathrm{msec})$ than on correct feedback $(756 \mathrm{msec})$ trials $(p<.001)$. Like the adults, adolescents had slower responses on error negative feedback trials $(1,004 \mathrm{msec})$ than on correct feedback $(877 \mathrm{msec})$ trials $(p<.001)$. Children also showed slower responses on error $(1,184 \mathrm{msec})$ than on correct $(1,121 \mathrm{msec})$ conditions $(p \mathrm{~s}<.025)$, but this effect was smaller than for the two older groups.

To summarize, over 300 trials, children made fewer rule shifts (as indicated by fewer first warning and first positive feedback observations), fewer efficient errors, and more performance errors. In contrast, adolescents' performance was highly similar to the performance of adults, although the adolescents showed a trend toward more performance errors. Both adolescents and adults slowed more on error trials (i.e., those followed by error feedback), relative to correct trials, than did children.

\section{Whole-Brain Analysis: \\ Feedback-Related Activation}

The whole-brain analysis modeled each trial as a single event, starting at the feedback display. Contrast images, computed separately for adults, adolescents, and children (Figure 4; see the supplementary materials for tables of activation for negative $>$ positive feedback [Table 1] and positive $>$ negative feedback [Table 2]) revealed several areas of activation related to processing negative relative to positive feedback. In adults, the negative $>$ positive feedback contrast resulted in activation in DLPFC (BA 9), medial prefrontal cortex/ACC (BAs 32, 6, 8), and superior and inferior parietal cortex (BAs 7, 40) $(p<.05$, corrected for multiple comparisons). Uncorrected for multiple comparisons $(p<.001)$, the negative $>$ positive feedback contrast also showed increased activation in bilateral lat-OFC (BA 47) extending into the insula (BA 13), as well as in anterior prefrontal cortex (BA 10).

The negative $>$ positive feedback contrast for adolescents resulted in overlapping areas of activation relative to adults. Increased activation for negative feedback was observed in lat-OFC/insula (BAs 47, 13), anterior prefrontal cortex (BA 10), medial PFC/ACC (BAs 32, 6, 8), and superior and inferior parietal cortex (BAs 7, 40) $(p<$ .05 , corrected for multiple comparisons). Uncorrected for multiple comparisons $(p<.001)$, activation was also significant in DLPFC (BAs 9, 46).

In children, considerably fewer areas of activation were revealed at the same threshold. In fact, no activations were apparent for the contrast negative $>$ positive feedback when corrected for multiple comparisons. Uncorrected for multiple comparisons ( $p<.001$, shown in Figure 4), the negative $>$ positive feedback contrast resulted in activation in the same regions as found in adolescents and adults, including

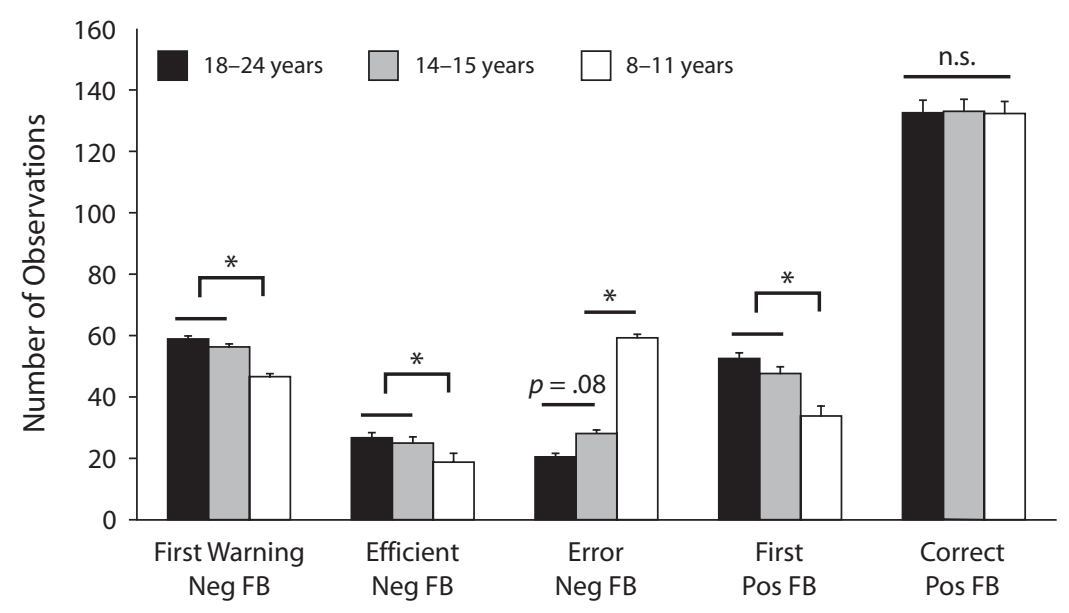

Figure 3. Performance as indicated by the number of feedback types. Children performed more poorly than adults and adolescents, as indicated by fewer first warning feedbacks (indicating rule shifts), fewer efficient errors, and more performance errors. 


\section{Negative FB > Positive FB}

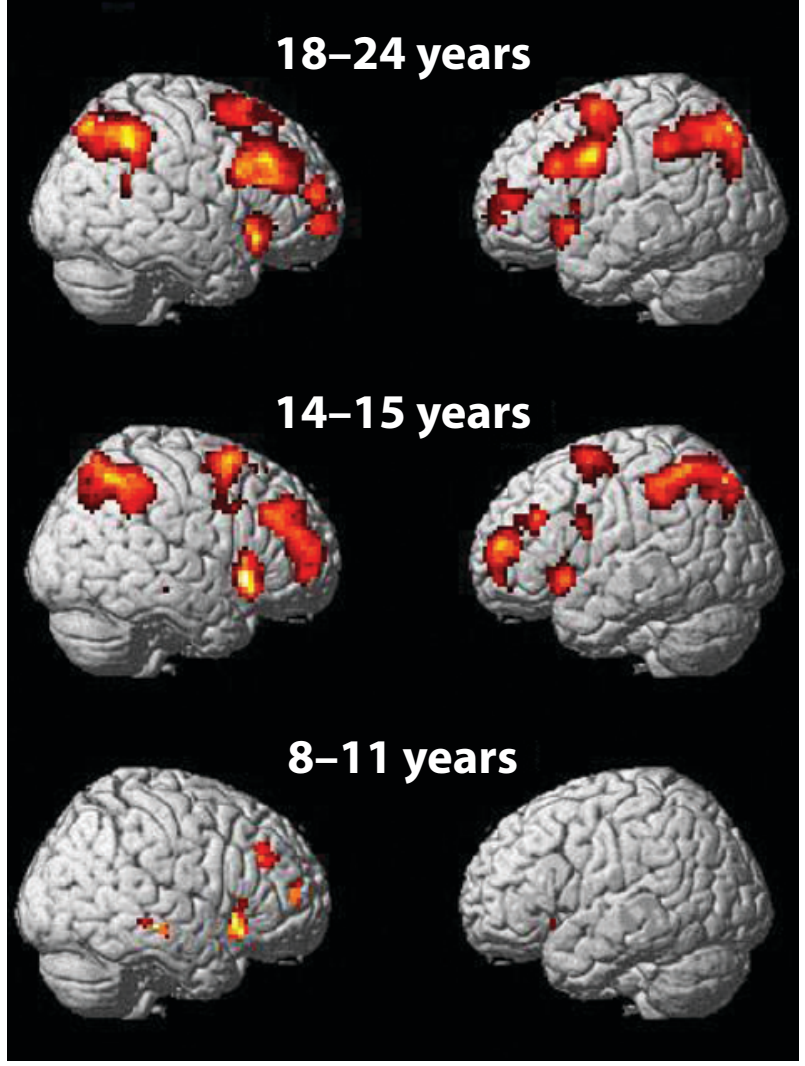

Figure 4. Feedback-locked whole-brain contrasts are displayed for 18- to 24-year-olds, 14- to 15-year-olds, and 8- to 11-year-olds, showing effects of negative feedback $>$ positive feedback at $p<$ .001 , uncorrected for multiple comparisons. Coordinates are reported in Supplementary Table 1.

DLPFC (BAs 9, 46), lat-OFC/insula (BAs 47, 13), anterior PFC (BA 10), and medial PFC/ACC (BAs 32, 6, 8).

Together, the whole-brain comparisons indicate that participants of all age groups recruited a network of overlapping brain regions when processing negative relative to positive feedback, including DLPFC, medial PFC/ACC, lat-OFC, and superior and inferior parietal cortex. We performed ROI analyses in order to examine the correlations between age, performance, and the neural activation associated with negative $>$ positive feedback. Next, we examined the pattern of activation in these regions for each feedback type.

\section{ROI Analyses}

ROI analyses were performed for right DLPFC, ACC, right lat-OFC, and right superior parietal cortex on the basis of an all feedback $>$ fixation contrast across participants. Analyses focused on regions within the right hemisphere because these condition effects were strongest there. For each ROI, we extracted activation levels relative to a fixation baseline for each condition and participant.

Brain-behavior correlations. Our first set of analyses focused on the correlations between age, performance, and differences in neural activation following negative relative to positive feedback. For this analysis, we computed the difference scores for the negative $>$ positive feedback contrast for each ROI. We correlated these scores with age, number of first warning errors, number of efficient errors, and number of erroneous responses. For right DLPFC and right superior parietal cortex, these analyses resulted in significant positive correlations with age $(r=.34, p<.009$, and $r=.32, p<.016)$, number of first warning errors $(r=.28$, $p<.034$, and $r=.43, p<.001$ ), and number of efficient errors $(r=.34, p<.01$, and $r=.27, p<.04)$. Thus, older individuals showed greater differentiation between negative and positive feedback, and more activation following negative feedback was associated with more first warning errors (indicating more rule switches) and more efficient errors (indicating successful feedback use). The same regions (right DLPFC and right superior parietal cortex) correlated negatively with the number of erroneous responses $(r=$ $-.36, p<.006$, and $r=-.42, p<.001)$. Thus, less activation was associated with more performance errors. All of these correlations were also significant for left DLPFC and superior parietal cortex (all $p \mathrm{~s}<.05$ ). In contrast, none of the correlations were significant for right and left ACC or for right and left OFC (all $p \mathrm{~s}>.15$ ).

To examine in more detail the neural patterns associated with the different feedback types (first warning feedback, efficient negative feedback, error feedback, first positive feedback, and correct feedback) across age groups, separate repeated measures ANOVAs for each region were performed.

DLPFC. The age groups $(3) \times$ feedback type (5) ANOVA for activation in DLPFC resulted in a main effect of feedback $[F(4,216)=34.12, p<.005]$, which was qualified by an age group $\times$ feedback interaction $[F(8,216)=2.58, p<.01]$. Separate comparisons indicated that the patterns of activation differed significantly between adults and adolescents $[F(4,152)=2.97, p<$ $.05]$ and between children and adults $[F(4,140)=3.71$, $p<.01]$, but not between adolescents and children $[F(4,140)=0.72, p=.58]$.

Analyses for each age group separately were performed to characterize the differential patterns of activation across age groups. As can be seen in Figure 5, in adults, all three negative feedback types resulted in more activation than did positive feedback (all $p s<.05$ ). Comparison of the three negative feedback conditions showed that error feedback resulted in increased activation relative to first warning feedback $[F(1,19)=30.85, p<.001]$, and activation associated with efficient negative feedback showed an intermediate pattern (the efficient negative feedback condition did not differ significantly from either the error feedback, $p=.09$, or the first warning feedback, $p=.12$, condition). The activation associated with first positive feedback and correct positive feedback did not differ $(p=.35)$. Similar analyses for the adolescent group demonstrated that adolescents also showed higher activation in DLPFC following all negative feedbacks relative to the positive feedbacks (all $p \mathrm{~s}<.05$; see Figure 5). However, unlike with adults, there were no differential patterns of activation following first warning, efficient, and error negative feedback 
$[F(2,38)=2.20, p=.12]$. In addition, first positive feedback resulted in more activation than did correct feedback $[F(1,19)=10.64, p<.005]$. Finally, comparisons for children also revealed that all negative feedback resulted in more activation than did positive feedback (all $p$ s $<.05$ ). Children differentiated between different types of negative feedback in such a way that both efficient negative feedback and error negative feedback resulted in more activation than did first warning negative feedback $(p<.01$ and $p<.05$, respectively), whereas efficient negative feedback and error negative feedback did not differ from each other $(p=.25)$. First positive and correct positive feedback also did not differ from each other $(p=.39)$.

To summarize, the patterns of activation in DLPFC differed between adults, adolescents, and children. All age groups showed increased activation following negative relative to positive feedback. Adults showed the highest activation for error feedback, followed by efficient feedback, followed by first warning feedback, indicating that DLPFC was sensitive to feedback that signaled the correct response. In contrast, for adolescents and children, there was less or no differentiation between the three negative feedback types, suggesting that these groups failed to differentiate between feedback that signaled errors and feedback that was informative for finding the correct rule.

Medial PFC/ACC. The age group $(3) \times$ feedback type (5) ANOVA for ACC also resulted in a main effect of feedback $[F(4,216)=26.43, p<.001]$ and an age group $\times$ feedback interaction $[F(8,216)=3.14, p<.005]$. Separate comparisons indicated that, as in DLPFC, the pattern of activation differed significantly between adults and adolescents $[F(4,152)=3.97, p<.005]$ and between children and adults $[F(4,140)=4.71, p<.001]$, but not between adolescents and children $[F(4,140)=0.62, p=.65]$.

The feedback (5) analysis for adults only demonstrated that first warning and error feedback resulted in increased activation relative to positive feedback (all $p \mathrm{~s}<.05$ ), and first positive and correct positive feedback did not differ from each other $(p=.97)$. The activation following error feedback was larger than that following first warning feedback $(p<.001)$. In contrast, efficient negative feedback did not differ from first positive or correct positive feedback (both $p \mathrm{~s}>.50$ ). The same analyses for adolescents, in contrast, revealed that there were no significant differences in the activation patterns following the three negative feedback types $[F(2,38)=2.45, p=.10]$, that efficient negative feedback resulted in higher activation than did first positive $(p<.005)$ and correct feedback $(p<.001)$, and that the two types of positive feedback did not differ from each other $(p=.58)$. The same comparison for the children revealed, as in adolescents, that there were no differences in the activation patterns following the three negative feedback types $[F(2,32)=2.38, p=.11]$ and that efficient negative feedback resulted in higher activation than did correct feedback $(p<.05)$, although the difference between efficient negative feedback and first positive feedback was not significant $(p=.19)$. First positive and correct feedback did not differ from each other $(p=.46)$.

The pattern of activation in medial PFC differed significantly from the pattern observed in DLPFC, as indicated by a significant age group $\times$ feedback $\times$ region interaction $[F(8,216)=2.00, p<.05]$. Post hoc ANOVAs for each age group separately confirmed region $\times$ feedback interactions for adults $[F(4,76)=9.14, p<.001]$ and adolescents $[F(4,76)=3.03, p<.05]$, but not for children $[F(4,64)=1.63, p=.17]$.

To summarize, for ACC the patterns of activation also differed between adults, adolescents, and children. For adults, ACC was most active following unexpected feedback (first warning feedback and error feedback), but not following anticipated negative feedback (efficient negative feedback). In contrast, adolescents and children failed to discriminate between the informative values of the types of negative feedback and showed increased activation following all three types.

Lat-OFC. The age group (3) $\times$ feedback (5) ANOVA for lat-OFC resulted in a significant feedback effect $[F(4,216)=44.46, p<.001]$, but this effect was not different between the age groups [age group $\times$ feedback interaction: $F(8,216)=0.94, p=.49]$. Post hoc comparisons demonstrated that lat-OFC was more active following error feedback than following first warning feedback $[F(1,54)=$ $9.51, p<.005]$. Efficient feedback resulted in an intermediate activation pattern that did not differ significantly from the activation for either first warning $(p=.08)$ or error $(p=.17)$ feedback. First positive and correct feedback did not differ from each other ( $p=.38)$, but their activation was significantly lower than for all negative feedback types (all $p \mathrm{~s}<.001$ ).

The differential age pattern for lat-OFC relative to DLPFC was confirmed by a significant age group $\times$ feedback $\times$ region interaction $[F(8,216)=2.96, p<.005]$. Post hoc ANOVAs for each age group separately revealed a nonsignificant region $\times$ feedback interaction for adults $[F(4,76)=1.11, p=.36]$ but significant interactions for adolescents $[F(4,76)=3.92, p<.005]$ and children $[F(4,64)=3.35, p<.05]$.

Together, these results demonstrate that for all age groups the pattern of activation in lat-OFC mirrored the activation pattern of DLPFC in adults. Errors resulted in the most activation, followed by efficient negative feedback, followed by first warning feedback, and all types of negative feedback resulted in more activation than did positive feedback.

Superior parietal cortex. The age group $(3) \times$ feedback (5) ANOVA for superior parietal cortex also resulted in a main effect of feedback $[F(4,216)=23.58, p<.001]$ and an age group $\times$ feedback interaction $[F(8,216)=$ $2.32, p<.05]$. Separate comparisons indicated that the patterns of activation differed significantly between adults and children $[F(4,140)=3.81, p<.01]$ and between adolescents and children $[F(4,140)=2.71, p<$ $.05]$, but not between adults and adolescents $[F(4,152)=$ $0.37, p=.83]$.

Separate comparisons for adults demonstrated that activation here did not differ between the three negative feedback types $[F(2,38)=1.06, p=.36]$. However, a comparison for the positive feedback types showed that activation following first positive feedback was higher than that following correct feedback $(p<.001)$. Activation following first positive feedback did not differ from that following 


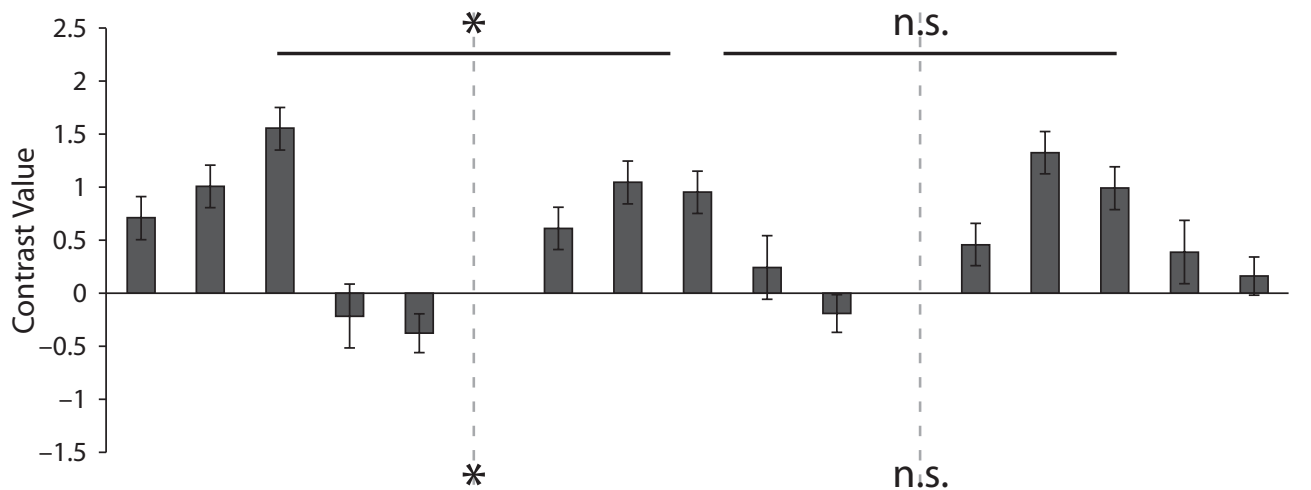

Right DLPFC (BA 9) $44,38,30$
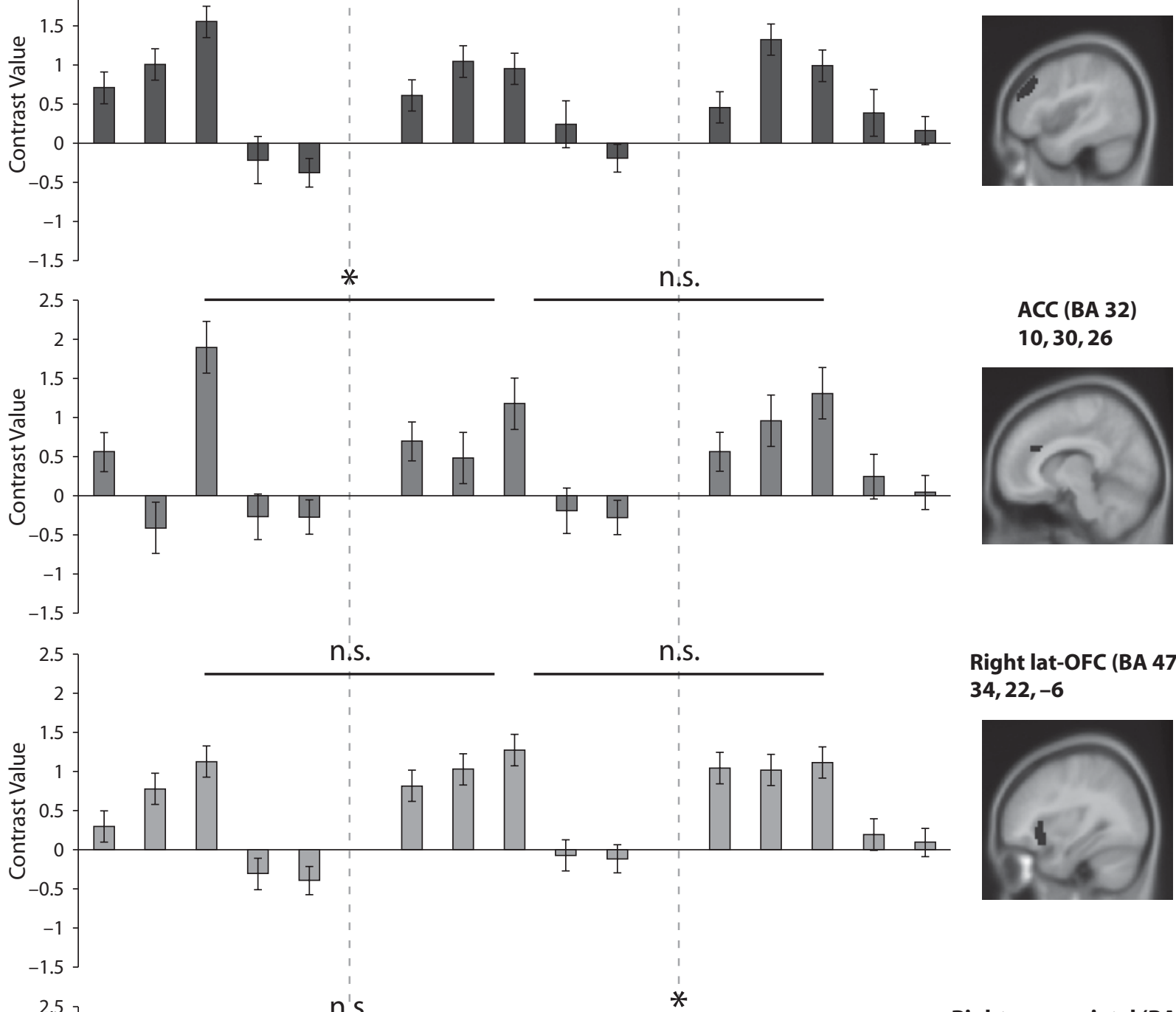

Right lat-OFC (BA 47) $34,22,-6$

ACC (BA 32)

$10,30,26$
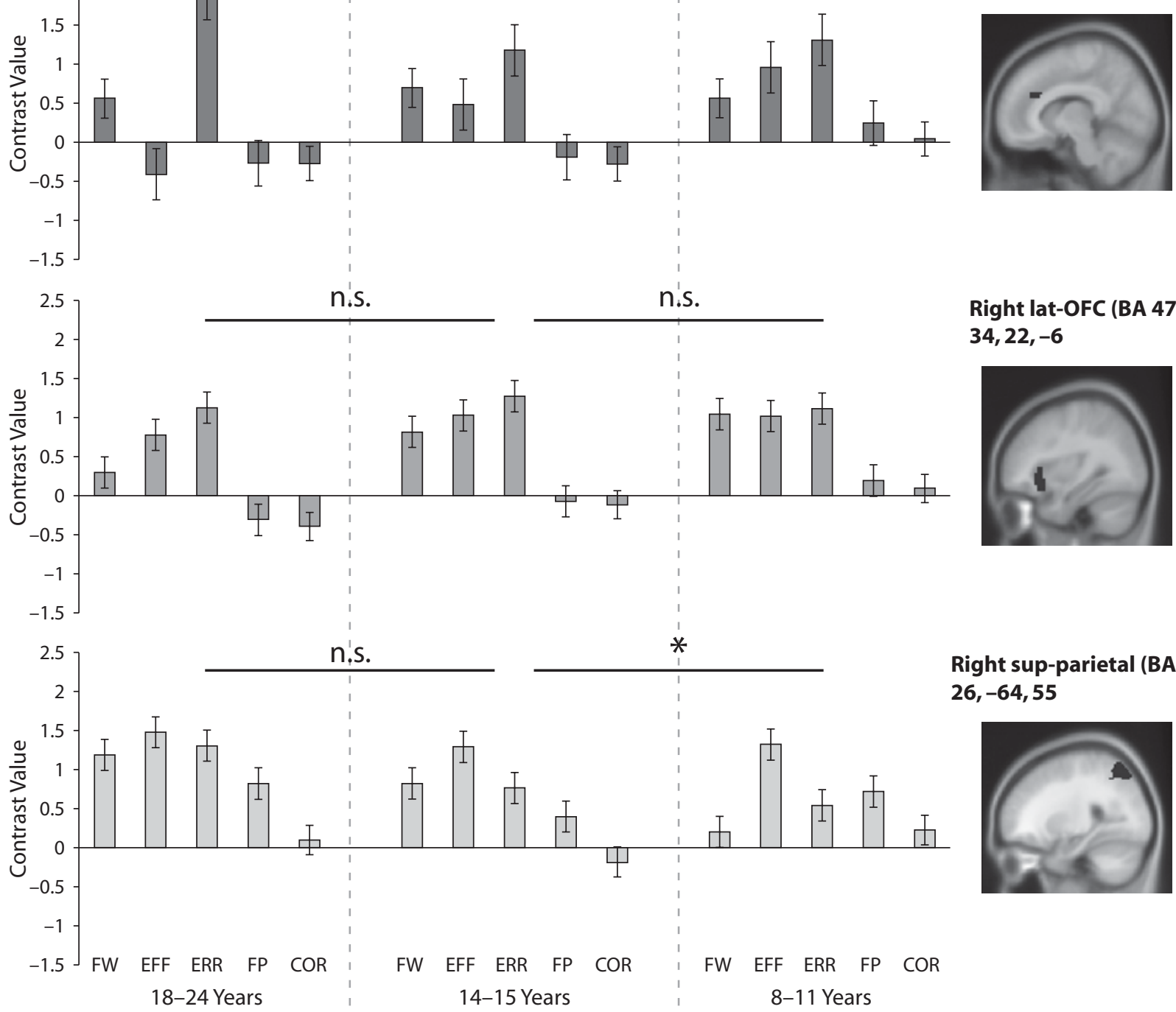

Right sup-parietal (BA 7) $26,-64,55$

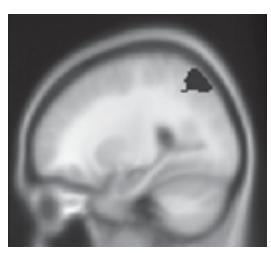

Figure 5. Region-of-interest activation values for dorsolateral prefrontal cortex (DLPFC), anterior cingulate cortex (ACC), lateral orbitofrontal cortex (lat-OFC), and superior parietal cortex for all three age groups. FW, first warning feedback; EFF, efficient negative feedback; ERR, error negative feedback; FP, first positive feedback; COR, correct positive feedback. " "Significant age $\times$ condition interaction, $p<.05$. 
first warning feedback ( $p=.13$ ) but was smaller than activation following efficient $(p<.005)$ and error negative $(p<.005)$ feedback. The same analyses for adolescents mirrored the results of adults, showing a nonsignificant differentiation between the three negative feedback types $[F(2,38)=2.70, p=.09]$ and increased activation following first positive feedback relative to correct positive feedback $(p<.001)$. First positive feedback resulted in lower activation than did either first warning $(p<.01)$ or efficient negative $(p<.001)$ feedback, but it did not differ from error feedback $(p=.20)$. The pattern of activation for children was different from those observed for adults and adolescents. Unlike in the older age groups, with children, activation in superior parietal cortex differentiated between the three negative feedback types $[F(2,32)=10.29, p<$ .001]. Efficient negative feedback resulted in increased activation relative to both first warning feedback $(p<.001)$ and error feedback $(p<.001)$, and the latter two did not differ from each other $(p=.28)$. First positive feedback resulted in a trend toward higher activation than following correct feedback ( $p=.07)$ and a trend toward lower activation than following efficient negative feedback $(p=.07)$, but it did not differ from first warning negative feedback $(p=.24)$ or error feedback $(p=.49)$.

A comparison of activation in superior parietal cortex relative to that in the frontal regions revealed that the age $X$ feedback $\times$ region interaction for superior parietal cortex relative to DLPFC was not significant $[F(8,216)=1.63$, $p=.11]$, although there were strong age group $\times$ feedback $[F(8,216)=2.68, p<.001]$ and region $\times$ feedback $[F(4,216)=10.02, p<.001]$ interactions. In contrast, the age $\times$ feedback $\times$ region interaction was significant for superior parietal cortex relative to both $\mathrm{ACC}[F(8,216)=$ $1.95, p<.05]$ and lat-OFC $[F(8,216)=2.16, p<.05]$, confirming that, over the course of development, these regions contributed differently to feedback processing.

To summarize, activation in superior parietal cortex only differed between children and adolescents and between children and adults, not between adolescents and adults. The patterns of activation demonstrated that adults and adolescents did not use superior parietal cortex to differentiate between the informative values of the types of negative feedback, although both groups did show increased activation following positive feedback, indicating that the correct rule had been found (first positive feedback). In contrast, children showed increased activation in this region following efficient negative feedback relative to the other negative feedback types, suggesting that they used this region to test hypotheses about rule use.

\section{DISCUSSION}

This developmental fMRI study was aimed at examining developmental differences in the neural correlates of feedback processing when switching between rules for correct behavior. On the basis of prior studies in adults, we focused on three regions within prefrontal cortex-OFC, ACC, and DLPFC - and also examined the role of superior parietal cortex. Differential developmental trajectories were found for these regions during childhood and adoles- cence. Our analyses revealed three main findings: (1) All regions were most sensitive to error negative feedback. However, the regions showed differential sensitivity to the other feedback types as well. DLPFC and superior parietal cortex were more sensitive to feedback that signaled the correct rule for subsequent trials (efficient negative or first positive feedback) - that is, these regions were important for hypothesis testing - whereas ACC was more sensitive to feedback signaling a violation of expectations (first warning feedback). (2) OFC, ACC, DLPFC, and superior parietal cortex had separate trajectories of functional maturation, with OFC maturing earliest, followed by superior parietal cortex; the slowest maturation was found for ACC and DLPFC. (3) Across the course of development, these regions contributed to feedback processing in different ways, with a shift in importance from superior parietal cortex in childhood to ACC and DLPFC in adulthood.

\section{Separable Brain Regions Contribute to Different Aspects of Feedback Processing in Adults}

We first examined whether adults recruited brain regions differently following different types of feedback (see also Zanolie et al., 2008). As expected, adults received approximately $50 \%$ efficient negative feedback, showing that they made successful use of the feedback (e.g., if Rule A was incorrect, then Rule B [ $50 \%$ chance] or Rule C [ $50 \%$ chance] must be correct). In addition, they slowed their responses on trials that were followed by error feedback, suggesting that errors resulted from effortful rule searching or attention slips. The fMRI results demonstrate that OFC, DLPFC, ACC, and superior parietal cortex contributed to feedback processing in different ways. All regions were most sensitive to feedback indicating an erroneous response, consistent with prior studies that have revealed that errors signal the demand for performance adjustment (Holroyd \& Coles, 2002). Erroneous responses are usually the result of actions when the participant could have known better, and therefore the neural response associated with this type of feedback could reflect prediction errors. Therefore, the neural activation associated with error feedback could also be the result of response-related activation just prior to the presentation of the feedback. It is likely that these errors (1) violate the participants' expectations and (2) result in an increased need for behavioral adjustment. We dissociated these two processes further by examining neural responses to first warning and efficient negative feedback. These feedback types demonstrated a dissociation between the involvement of DLPFC and ACC. That is, DLPFC was more active following efficient than following first warning negative feedback, whereas ACC was more active following first warning than following efficient negative feedback. We interpreted these results as indicating that DLPFC signaled the need for a change in goal-directed behavior (i.e., efficient negative feedback constrained future action possibilities and signaled the correct rule for the next trial). In contrast, ACC is thought to be sensitive to feedback that violates expectations and signals uncertainty (first warning of rule change; Zanolie et al., 2008).

Superior parietal cortex was more active following error and efficient negative feedback, relative to first warning 
feedback. In addition, this region was also sensitive to first positive feedback. This pattern of activation shows that superior parietal cortex is also sensitive to the informative value of the feedback, resembling the activation pattern found in DLPFC. Both efficient negative feedback and first positive feedback are used to find the correct rule for future trials, and therefore these feedbacks are thought to be important for hypothesis testing. Moreover, superior parietal cortex showed a strong correlation with successful rule shifting (as indexed by the number of first warning feedbacks), strengthening our hypothesis that superior parietal cortex is also important for informative feedback processing. To our knowledge, this is the first study reporting on the role of superior parietal cortex in feedback processing. Our results are consistent with prior studies that have demonstrated that parietal cortex is important for working memory (Klingberg et al., 2002; Ravizza, Delgado, Chein, Becker, \& Fiez, 2004) and higher order cognition (Gray, Chabris, \& Braver, 2003).

One alternative explanation should be taken into account. As can be seen in Figure 3, the different feedback types occurred with different frequencies. Therefore, some of our effects may have been influenced by (in)frequency effects. For ACC, this explanation is unlikely, because (at least in adults) this region was more active following first warning negative feedback than following efficient negative feedback, even though first warning negative feedbacks occurred with a higher frequency. However, for DLPFC and superior parietal cortex, regions that were most active following feedback types that occurred with lower frequencies (errors, efficient negative feedback, or first positive feedback), it is possible that part of their responsiveness was the result of a greater response to infrequently occurring stimuli. This explanation is not necessarily inconsistent with the idea that these regions are sensitive to stimuli signaling hypothesis testing; infrequent stimuli may be more salient, and therefore provide important cues for testing whether the current behavioral response is correct. In the present paradigm, we could not dissociate frequency effects from the importance of signals for hypothesis testing, but this issue could be the focus of future research. In this study, it was most important that the ACC, DLPFC, and parietal cortex contributed to feedback processing in different ways.

Lat-OFC was also more active following negative rather than positive feedback, consistent with prior studies showing that this region is sensitive to loss (O'Doherty et al., 2003). Activation in lat-OFC did not differ from that in DLPFC. Possibly, lat-OFC in the present design was more sensitive to learning, given that there was no money at stake.

\section{Separate Developmental Trajectories for OFC, ACC, DLPFC, and Parietal Cortex}

The findings of this study show clear developmental differences in the trajectories of functional maturation in lat-OFC, superior parietal cortex, DLPFC, and ACC. For lat-OFC, there were no differences in activation pattern between age groups, and whole-brain comparisons indicated consistent activation for the negative $>$ positive contrast in all age groups. This finding is consistent with prior studies that have suggested that lat-OFC is sensitive to the valence of feedback (Frank \& Claus, 2006; O’Doherty et al., 2003), and this sensitivity may be similar for all age groups. Indeed, prior studies that have examined the role of lat-OFC have suggested that activation in this region is greater for children than for adults, either because children may experience negative outcomes as more aversive or because negative feedback is unexpected (Galvan et al., 2006; Van Leijenhorst et al., 2006). These effects may be more pronounced in gambling paradigms or in paradigms in which participants make decisions under uncertainty, when the function of the valence of feedback is more complex. In the present paradigm, valence effects may have been similar across age groups, since positive or negative feedback simply served as a signal that behavior was to be continued or changed.

Superior parietal cortex activation in 8- to 11-year-olds differed from that in adults, but in adolescents it showed an adult pattern. In contrast, DLPFC and ACC showed an activation pattern that was still not mature in adolescents. This result is consistent with an earlier study in which we found that superior parietal cortex matured earlier than ventrolateral prefrontal cortex in a task-switching paradigm (Crone, Donohue, Honomichl, Wendelken, \& Bunge, 2006). Structural brain imaging studies of development have indicated late gray matter loss and white matter increases during late childhood and adolescence, developments associated respectively with the pruning of excessive neurons and increased structural connectivity between regions (Gogtay et al., 2004). Longitudinal measurements have indicated that cortical gray matter structure is mature earlier for $\mathrm{OFC}$ than for superior parietal cortex, DLPFC, and ACC (Sowell et al., 2004), lending support to our findings of functional maturation in these regions. The differential developmental trajectories for superior parietal cortex relative to DLPFC and ACC may result in children using different strategies to perform the task. This possibility is described in more detail below.

\section{Functional Differences in Activation Patterns Across Development}

Consistent with prior studies (Huizinga et al., 2006; Welsh et al., 1991), our behavioral comparisons demonstrated pronounced differences in the task performance of children relative to adolescents and adults. Children encountered fewer rule shifts, made fewer efficient errors, and made more errors in general when performing the task. Given that participants performed a fixed number of trials, on the basis of the present behavioral data it cannot be determined whether children were less successful in rule shifting following first warning errors or whether they failed to maintain the currently relevant rule online (for a discussion of these two interpretations, see Crone, Ridderinkhof, Worm, Somsen, \& van der Molen, 2004). The behavioral changes between groups were accompanied by changes in activation patterns in ACC, DLPFC, and superior parietal cortex. In contrast to adults, children did not use ACC and DLPFC to differentiate between the informative values of negative feedback. Instead, these regions showed a general sensitivity to all negative feedback. Children were successful in using superior parietal cortex to differentiate between the 
informative values of feedback, and this differential pattern was larger for children than for adults.

These results demonstrate two important points: First, children may use a different strategy than older groups when performing this task; in particular, they may fail to develop hypotheses when searching for the correct rule (Somsen, 2007). All age groups showed more activation in ACC and DLPFC following negative feedback, but adults used ACC more to indicate an expectation violation and DLPFC more to employ goal-directed actions when possibilities were constrained. In contrast, children showed similar activation in these regions following all types of negative feedback. Thus, they may not have experienced the feedback as signaling different informative values. This assumption is reinforced by the RT results, which showed that adults and adolescents slowed more than children on trials followed by error feedback. This interpretation is also consistent with an earlier study in which children showed a heart rate response to all types of negative feedback, both informative and uninformative, whereas adults only showed heart rate slowing following informative negative feedback (Crone, Jennings, \& van der Molen, 2004).

Second, children may use a compensatory network to perform the task, by relying more on parietal cortex than do adults. This assumption is demonstrated by the finding that children show more activation following efficient negative feedback and first positive feedback in this region, relative to adults. Thus, children may search for the correct rule by increased activation in parietal cortex during the rule search process. This interpretation is consistent with the assumption that over the course of development, cortical regions become more fine-tuned to perform tasks in the most efficient way (Casey, Tottenham, Liston, \& Durston, 2005).

Despite the absence of performance differences, adolescents still differed from adults in brain activation in ACC and DLPFC. In contrast, in adolescence the pattern of activation in superior parietal cortex was already at adult level (see also Casey et al., 2005). In ACC and DLPFC, the activation pattern of 14- to 15-year-old adolescents did not differ from that of 8- to 11-year-old children. ERP studies focusing on error monitoring without feedback in adolescence have also demonstrated adultlike performance but smaller ERN potentials in adolescents following an erroneous response (Davies et al., 2004; Ladouceur, Dahl, \& Carter, 2004). This result is important, because it underscores the fact that differences may seem absent on the basis of behavior in a structured experimental setting, but they may still be present in brain activation. This result is especially important in adolescent research, because adolescents may seem mature in terms of cognitive development but are often immature in terms of planning future actions. The results of this study show that this may result from late maturation of brain regions that are important for flexible performance adjustment following changing task demands (see also Luna \& Sweeney, 2004).

In conclusion, our fMRI results provide evidence for separable neurodevelopmental trajectories for brain regions that have been implicated in feedback processing in adults. Unlike 8- to 11-year-old children and 14- to 15-year-old adolescents, adults used ACC and DLPFC differently in response to negative performance feedback that signaled information related to expectation violation or goal-directed actions. These findings may contribute to the developmental differences in performance in 8- to 11 -year-olds. The brain differences were also evident in 14- to 15-year-old adolescents, despite their performance being similar to that of adults. Furthermore, 8- to 11-yearolds, but not adolescents, showed a differential pattern of activation in superior parietal cortex relative to adults, suggesting that children may have used superior parietal cortex as a compensatory region. Developmental changes in feedback processing may result from increased specialization in brain regions that contribute to separate aspects of performance monitoring and from increased reliance on prefrontal cortex relative to parietal cortex regions.

\section{AUTHOR NOTE}

The authors thank Wouter Teeuwisse for technical assistance. Research from the first and last authors (E.A.C. and S.A.R.B.R.) is made possible by NWO VENI/VIDI grants. Correspondence relating to this article may be sent to E. A. Crone, Leiden University, Department of Developmental Psychology, Wassenaarseweg 52, 2333AK Leiden, The Netherlands (e-mail: ecrone@fsw.leidenuniv.nl).

\section{REFERENCES}

ACHENBACH, T. M. (1991). Manual for the Child Behavior Checklist/4-18 and 1991 profile. Burlington: University of Vermont, Department of Psychiatry.

Barceló, F., \& KNight, R. T. (2002). Both random and perseverative errors underlie WCST deficits in prefrontal patients. Neuropsychologia, 40, 349-356.

Bechara, A., Damasio, H., \& Damasio, A. R. (2000). Emotion, decision making and the orbitofrontal cortex. Cerebral Cortex, 10, 295-307.

Brett, M., Anton, J.-L., Valabregue, R., \& Poline, J.-B. (2002, June). Region of interest analysis using an SPM toolbox. Paper presented at the 8th International Conference on Functional Mapping of the Human Brain, Sendai, Japan.

Bunge, S. A., Hazeltine, E., Scanlon, M. D., Rosen, A. C., \& GABRIELI, J. D. E. (2002). Dissociable contributions of prefrontal and parietal cortices to response selection. NeuroImage, 17, 1562-1571.

Casey, B. J., Tottenham, N., Liston, C., \& Durston, S. (2005). Imaging the developing brain: What have we learned about cognitive development? Trends in Cognitive Sciences, 9, 104-110.

Cocosco, C. A., Kollokian, V., Kwan, R. K.-S., \& Evans, A. C. (1997). BrainWeb: Online interface to a 3D MRI simulated brain database. Neurolmage, 5, S425.

Crone, E. A., Donohue, S. E., Honomichl, R., Wendelken, C., \& Bunge, S. A. (2006). Brain regions mediating flexible rule use during development. Journal of Neuroscience, 26, 11239-11247.

Crone, E. A., Jennings, J. R., \& van der Molen, M. W. (2004). Developmental change in feedback processing as reflected by phasic heart rate changes. Developmental Psychology, 40, 1228-1238.

Crone, E. A., Ridderinkhof, K. R., Worm, M., Somsen, R. J. M., \& VAN DER MOLEN, M. W. (2004). Switching between spatial stimulusresponse mappings: A developmental study of cognitive flexibility. Developmental Science, 7, 443-455.

Crone, E. A., Somsen, R. J. M., Zanolie, K., \& van der Molen, M. W. (2006). A heart rate analysis of developmental change in feedback processing and rule shifting from childhood to early adulthood. Journal of Experimental Child Psychology, 95, 99-116.

Crone, E. A., van der Veen, F. M., van der Molen, M. W., Somsen, R. J. M., van Beek, B., \& Jennings, J. R. (2003). Cardiac concomitants of feedback processing. Biological Psychology, 64, 143-156.

Crone, E. A., Wendelken, C., Donohue, S., Van Leijenhorst, L., $\&$ Bunge, S. A. (2006). Neurocognitive development of the ability to manipulate information in working memory. Proceedings of the National Academy of Sciences, 103, 9315-9320. 
Dale, A. M. (1999). Optimal experimental design for event-related fMRI. Human Brain Mapping, 8, 109-114.

Davies, P. L., Segalowitz, S. J., \& Gavin, W. J. (2004). Development of error-monitoring event-related potentials in adolescents. In R. E. Dahl \& L. P. Spear (Eds.), Adolescent brain development: Vulnerabilities and opportunities (Annals of the New York Academy of Sciences, Vol. 1021, pp. 324-328). New York: New York Academy of Sciences.

Demakis, G. J. (2003). A meta-analytic review of the sensitivity of the Wisconsin Card Sorting Test to frontal and lateralized frontal brain damage. Neuropsychology, 17, 255-264.

Falkenstein, M., Hohnsbein, J., Hoormann, J., \& Blanke, L. (1991). Effects of crossmodal divided attention on late ERP components: II. Error processing in choice reaction tasks. Electroencephalography \& Clinical Neurophysiology, 78, 447-455.

Frank, M. J., \& Claus, E. D. (2006). Anatomy of a decision: Striatoorbitofrontal interactions in reinforcement learning, decision making, and reversal. Psychological Review, 113, 300-326.

Galvan, A., Hare, T. A., Parra, C. E., Penn, J., Voss, H., Glover, G., \& CASEY, B. J. (2006). Earlier development of the accumbens relative to orbitofrontal cortex might underlie risk-taking behavior in adolescents. Journal of Neuroscience, 26, 6885-6892.

Gogtay, N., Giedd, J. N., Lusk, L., Hayashi, K. M., Greenstein, D., VAITUZIS, A. C., ET AL. (2004). Dynamic mapping of human cortical development during childhood through early adulthood. Proceedings of the National Academy of Sciences, 101, 8174-8179.

Gray, J. R., Chabris, C. F., \& Braver, T. S. (2003). Neural mechanisms of general fluid intelligence. Nature Neuroscience, 6, 316-322.

Heaton, R. K., Chelune, G. J., Talley, J. L., Kay, G. C., \& CuRTISS, G. (1993). Wisconsin Card Sorting Test manual: Revised and expanded. Odessa, FL: Psychological Assessment Resources.

Holroyd, C. B., \& Coles, M. G. H. (2002). The neural basis of human error processing: Reinforcement learning, dopamine, and the errorrelated negativity. Psychological Review, 109, 679-709.

Holroyd, C. B., Nieuwenhuis, S., Yeung, N., Nystrom, L., Mars, R. B., Coles, M. G. H., \& Cohen, J. D. (2004). Dorsal anterior cingulate cortex shows fMRI response to internal and external error signals. Nature Neuroscience, 7, 497-498.

Huizinga, M., Dolan, C. V., \& van Der Molen, M. W. (2006). Agerelated change in executive function: Developmental trends and a latent variable analysis. Neuropsychologia, 44, 2017-2036.

Kim, E. Y., Iwaki, N., Imashioya, H., Uno, H., \& Fujita, T. (2007). Error-related negativity in a visual go/no-go task: Children vs. adults. Developmental Neuropsychology, 31, 181-191.

Klingberg, T., Forssberg, H., \& Westerberg, H. (2002). Increased brain activity in frontal and parietal cortex underlies the development of visuospatial working memory capacity during childhood. Journal of Cognitive Neuroscience, 14, 1-10.

Knutson, B., Fong, G. W., Bennett, S. M., Adams, C. M., \& HomMER, D. (2003). A region of mesial prefrontal cortex tracks monetarily rewarding outcomes: Characterization with rapid event-related fMRI. Neurolmage, 18, 263-272.

Ladouceur, C. D., Dahl, R. E., \& CARTer, C. S. (2004). ERP correlates of action monitoring in adolescence. In R. E. Dahl \& L. P. Spear (Eds.), Adolescent brain development: Vulnerabilities and opportunities (Annals of the New York Academy of Sciences, Vol. 1021, pp. 329-336). New York: New York Academy of Sciences.

Liston, C., Matalon, S., Hare, T. A., Davidson, M. C., \& Casey, B. J. (2006). Anterior cingulate and posterior parietal cortices are sensitive to dissociable forms of conflict in a task-switching paradigm. Neuron, 50, 643-653.

Luciana, M., \& Nelson, C. A. (1998). The functional emergence of prefrontally-guided working memory systems in four- to eight-yearold children. Neuropsychologia, 36, 273-293.

LUNA, B., \& SWEENEY, J. A. (2004). The emergence of collaborative brain function: fMRI studies of the development of response inhibition. In R. E. Dahl \& L. P. Spear (Eds.), Adolescent brain development: Vulnerabilities and opportunities (Annals of the New York Academy of Sciences, Vol. 1021, pp. 296-309). New York: New York Academy of Sciences.

Mars, R. B., Coles, M. G. H., Grol, M. J., Holroyd, C. B., Nieuwenhuis, S., HulstiJn, W., \& Toni, I. (2005). Neural dynamics of error processing in medial frontal cortex. Neurolmage, 28, 1007-1013.

Miller, E. K., \& Cohen, J. D. (2001). An integrative theory of prefrontal cortex function. Annual Review of Neuroscience, 24, 167-202.
Milner, B. (1963). Effects of different brain regions on card sorting: The role of the frontal lobes. Archives of Neurology, 9, 90-100.

Monchi, O., Petrides, M., Petre, V., Worsley, K., \& Dagher, A. (2001). Wisconsin Card Sorting revisited: Distinct neural circuits participating in different stages of the task identified by event-related functional magnetic resonance imaging. Journal of Neuroscience, 21, 7733-7741.

Nieuwenhuis, S., Slagter, H. A., von Geusau, N. J. A., Heslenfeld, D. J., \& Holroyd, C. B. (2005). Knowing good from bad: Differential activation of human cortical areas by positive and negative outcomes. European Journal of Neuroscience, 21, 3161-3168.

O’Doherty, J., Critchley, H., Deichmann, R., \& Dolan, R. J. (2003). Dissociating valence of outcome from behavioral control in human orbital and ventral prefrontal cortices. Journal of Neuroscience, 23, 7931-7939.

Ravizza, S. M., Delgado, M. R., Chein, J. M., Becker, J. T., \& Fiez, J. A. (2004). Functional dissociations within the inferior parietal cortex in verbal working memory. Neurolmage, 22, 562-573.

Rizzolatti, G., LupPino, G., \& Matelli, M. (1998). The organization of the cortical motor system: New concepts. Electroencephalography \& Clinical Neurophysiology, 106, 283-296.

Rolls, E. T. (2004). The functions of the orbitofrontal cortex. Brain \& Cognition, 55, 11-29.

SomsEn, R. J. M. (2007). The development of attention regulation in the Wisconsin Card Sorting Task. Developmental Science, 10, 664-680.

Sowell, E. R., Thompson, P. M., Leonard, C. M., Welcome, S. E., KAN, E., \& TogA, A. W. (2004). Longitudinal mapping of cortical thickness and brain growth in normal children. Journal of Neuroscience, 24, 8223-8231.

TAlairach, J., \& Tournoux, P. (1988). Co-planar stereotaxic atlas of the human brain: 3-dimensional proportional system. An approach to cerebral imaging (M. Rayport, Trans.). Stuttgart: Thieme.

Van Leijenhorst, L., Crone, E. A., \& Bunge, S. A. (2006). Neural correlates of developmental differences in risk estimation and feedback processing. Neuropsychologia, 44, 2158-2170.

van Veen, V., Holroyd, C. B., Cohen, J. D., Stenger, V. A., \& CARTER, C. S. (2004). Errors without conflict: Implications for performance monitoring theories of anterior cingulate cortex. Brain \& Cognition, 56, 267-276.

Walton, M. E., Devlin, J. T., \& Rushworth, M. F. S. (2004). Interactions between decision making and performance monitoring within prefrontal cortex. Nature Neuroscience, 7, 1259-1265.

Welsh, M. C., Pennington, B. F., \& Groisser, D. B. (1991). A normativedevelopmental study of executive function: A window on prefrontal function in children. Developmental Neuropsychology, 7, 131-149.

Wiersema, J. R., van der Meere, J. J., \& Roeyers, H. (2007). Developmental changes in error monitoring: An event-related potential study. Neuropsychologia, 45, 1649-1657.

Zanolie, K., Van LeiJenhorst, L., Rombouts, S. A. R. B., \& Crone, E. A. (2008). Separable neural mechanisms contribute to feedback processing in a rule-learning task. Neuropsychologia, 46, 117-126.

\section{ARCHIVED MATERIALS}

The following materials associated with this article may be accessed through the Psychonomic Society's Norms, Stimuli, and Data archive, www.psychonomic.org/archive.

To access these files, search the archive for this article using the journal name (Cognitive, Affective, \& Behavioral Neuroscience), the first author's name (Crone), and the publication year (2008).

FILE: Crone-CABN-2008.zip

DESCRIPTION: The compressed archive file contains four files:

CroneAPP-A.txt, containing Supplementary Table 1.

CroneAPP-A.xls, containing the above information in Excel spreadsheet format.

CroneAPP-B.txt, containing Supplementary Table 2.

CroneAPP-B.xls, containing the above information in Excel spreadsheet format.

AUTHOR's E-MAIL ADDRESS: ecrone@fsw.leidenuniv.nl.

(Manuscript received September 6, 2007; revision accepted for publication January 1, 2008.) 